\title{
The structure and stability of molecular cloud cores in external radiation fields
}

\author{
D. Galli ${ }^{1}$, M. Walmsley ${ }^{1}$, and J. Gonçalves ${ }^{1,2}$ \\ 1 INAF-Osservatorio Astrofisico di Arcetri, Largo E. Fermi 5, 50125 Firenze, Italy \\ ${ }^{2}$ Centro de Astronomia e Astrofísica da Universidade de Lisboa, Tapada da Ajuda, 1349-018 Lisboa, Portugal
}

Received 31 May 2002 / Accepted 1 August 2002

\begin{abstract}
We have considered the thermal equilibrium in pre-protostellar cores in the approximation where the dust temperature is independent of interactions with the gas and where the gas is heated both by collisions with dust grains and ionization by cosmic rays. We have then used these results to study the stability of cores in hydrostatic equilibrium in the limit where thermal pressure dominates over magnetic field and turbulence. We compare the density distribution derived in this manner with results obtained in the isothermal case. We find that for cores with characteristics similar to those observed in nearby molecular clouds, the gas and dust temperatures are coupled in the core interior with densities above $\sim 3 \times 10^{4} \mathrm{~cm}^{-3}$. As a consequence, one expects that the gas temperature like the dust temperature decreases towards the center of these objects. However, the regime where gas and dust temperatures are coupled coincides approximately with that in which $\mathrm{CO}$ and many other molecular species deplete onto dust grain surfaces. At larger radii and lower densities, the gas and dust temperatures decouple and the gas temperature tends to the value expected for cosmic ray heating alone. The density structure which one computes taking into account such deviations from isothermality are not greatly different from that expected for an isothermal Bonnor-Ebert sphere. It is impossible in the framework of these models to have a stable equilibrium core with mass above $\sim 5 M_{\odot}$ and column density compatible with observed values $\left(N_{\mathrm{H}}>2 \times 10^{22} \mathrm{~cm}^{-2}\right.$ or $\left.A_{\mathrm{V}}>10 \mathrm{mag}\right)$. We conclude from this that observed high mass cores are either supported by magnetic field or turbulence or are already in a state of collapse. Lower mass cores on the other hand have stable states where thermal pressure alone provides support against gravitation and we conclude that the much studied object B68 may be in a state of stable equilibrium if the internal gas temperature is computed in self-consistent fashion. Finally we note that in molecular clouds such as Ophiuchus and Orion with high radiation fields and pressures, gas and dust temperatures are expected to be well coupled and hence in the absence of an internal heat source, one expects temperatures to decrease towards core centers and to be relatively high as compared to low pressure clouds like Taurus.
\end{abstract}

Key words. ISM: clouds, dust, extinction

\section{Introduction}

It has long been presumed that during the formation of a star, there is an intermediate phase in which the "protostar" is at least approximately in a state of hydrostatic equilibrium or magneto-hydrostatic equilibrium (see e.g. Shu et al. 1987). While this idea in origin was merely based on plausibility arguments, it has received support from the discovery that in least some nearby molecular clouds, one finds embedded "cores" of higher density than the surroundings where the observed linewidths are thermally dominated. That is to say, while there may be local subsonic turbulence, there is no evidence for collapse onto a point mass and it appears that thermal pressure is capable of balancing gravity. On the other hand, comparisons of the gravitational, thermal, magnetic and turbulent energies of such cores show that all these quantities are equal to within the (considerable) uncertainties (Myers \& Goodman 1988a,b;

Send offprint requests to: M. Walmsley, e-mail: walmsley@arcetri.astro.it
Myers et al. 1991; Crutcher 1999). These data suggest though they do not prove the existence of equilibrium structures which are an intermediate state in the evolution of a prestellar core.

This picture has received more observational support with the advent of high quality maps of the millimeter continuum emission of the dust grains within such cores as well as the possibility to study their extinction in the near and mid infrared (Johnstone et al. 2000, 2001; Bacmann et al. 2000; Lada et al. 1994). These have allowed a more unbiased view to be obtained of the density distribution in prestellar cores. In particular, they have shown that the early molecular line data was highly biased because above a critical density of $\sim 5 \times 10^{4} \mathrm{~cm}^{-3}$ (see Tafalla et al. 2002; Caselli et al. 2002a,b; Kramer et al. 1999) most molecular tracers including CO condense out onto dust grain surfaces. This depletion has the consequence that in molecular lines one sees mainly a lower density outer shell, whereas the dust emission (or absorption) offers a more unbiased view of the density distribution. 
The results from the millimeter continuum and infrared absorption studies have been compared with a variety of theoretical models of hydrostatic cores (Bacmann et al. 2000). One result of such studies has been evidence for a "flattening" in the density distribution for radii below a critical value of $r_{\mathrm{cr}} \simeq 2000-8000 \mathrm{AU}$ implying a steeper fall off in radius above $r_{\mathrm{cr}}$. Thus for example, Bacmann et al. (2000) model L1544 with a roughly uniform density inside 1900 AU but a rapid fall off outside this radius. Structures whose support against gravitational collapse is mainly due to the magnetic field are plausible both because the observed dust continuum maps show large departures from spherical symmetry and because of time scale arguments. Collapse on a free fall time scale would produce a larger star formation rate than that observed.

There are however some cores where thermal pressure may dominate magnetic pressure and spherical symmetry may be a good assumption. Particularly worthy of note is B68 where Alves et al. (2001) have recently demonstrated that the density distribution derived from their NIR measurements is consistent with a purely thermally supported hydrostatic model. In fact, they find results consistent with the equilibrium structures discussed by Ebert (1955) and Bonnor (1956), which we will refer to in the following as Bonnor-Ebert spheres. To what extent B68 is exceptional is presently unclear but we note that a large number of cores in the Ophiuchus and Orion clouds (Johnstone et al. 2000, 2001) appear to have characteristics compatible with Bonnor-Ebert spheres at temperatures of $15-30 \mathrm{~K}$.

This accord between theoretical expectation and observation suggests that a study of the theoretical assumptions may be worthwhile. One of these assumptions has been that of isothermality which is often based on the concept of "low optical depth for the cooling transitions". In reality, the gas in prestellar cores is thought to be cooled mainly by optically thick CO transitions (Goldsmith 2001) although, as mentioned above, the CO seems to disappear at high densities. Moreover, the typical density for which dust grains and gas become thermally coupled is roughly of the same order as that observed in prestellar cores (see, e.g. Krügel \& Walmsley 1984). Thus, we decided that a new look at the gas temperature distribution to be expected in such cores seemed warranted.

In this paper we replace the assumption of an isothermal gas with the more realistic condition of thermal balance in the gas, and we evaluate the consequences of an external heating source (the interstellar radiation field) on the structure of the cloud (density and temperature profiles) and its stability properties. Recent studies of the dust temperature distribution in such objects (Evans et al. 2001; Zucconi et al. 2001) have shown that the dust temperature typically falls by a factor of 2 from edge to center. It seems reasonable to ask how the gas temperature will react in such circumstances. One might also ask whether the density distribution in hydrostatic equilibrium will depart appreciably from that expected under the isothermal assumption. Will the expected density contrast differ for example from that expected for a Bonnor-Ebert sphere when one calculates the gas temperature in self-consistent fashion? This article represents an attempt to answer such questions.

The outline of this paper is as follows. In Sect. 2 we give a brief introduction to the theory of structures in hydrostatic equilibrium including the results for an isothermal equilibrium Bonnor-Ebert sphere. In Sect. 3 we discuss the input to our calculations and the simplifications which we have made. In Sect. 4, we present our results for the gas temperature distribution in two model cores whose density distribution has been assumed similar to that observed in L1544 and B68. In Sect. 5 we present our results for non-isothermal hydrostatic equilibria for a variety of conditions. Here we show among other things that the equilibria obtained depend relatively sensitively on the external radiation field. In Sect. 6 we discuss the observational consequences of our results, and compare the properties of our model clouds to isentropic polytropes. In Sect. 7 we summarize our conclusions.

\section{Theoretical background}

An isothermal gas sphere embedded in an external medium of given pressure $p_{\text {ext }}$ has a critical Bonnor-Ebert mass above which no state of equilibrium can exist (Ebert 1955; Bonnor 1956; McCrea 1957),

$$
\begin{aligned}
M_{\mathrm{BE}} & \simeq 1.182 \frac{a^{4}}{\sqrt{G^{3} p_{\mathrm{ext}}}} \\
& \simeq 2.6\left(\frac{T_{\mathrm{g}}}{10 \mathrm{~K}}\right)^{2}\left(\frac{p_{\mathrm{ext}}}{2 \times 10^{4} \mathrm{~K} \mathrm{~cm}^{-3}}\right)^{-1 / 2} M_{\odot},
\end{aligned}
$$

where $a=\left(k T_{\mathrm{g}} / \mu m_{\mathrm{H}}\right)^{1 / 2}$ is the sound speed in the gas, $T_{\mathrm{g}}$ the gas temperature and $\mu$ the mean molecular weight (see Fig. 2).

Below this critical value, the equation of hydrostatic equilibrium admits single or multiple solutions, characterized by different degrees of density concentration. It has been shown by Bonnor (1956) and Ebert (1957) that equilibrium configurations with center-to-boundary density contrast $\rho_{\mathrm{c}} / \rho_{\mathrm{b}}<13.98$ are stable, whereas equilibrium solutions with $\rho_{\mathrm{c}} / \rho_{\mathrm{b}}>13.98$ are unstable to radial collapse. Stable isothermal equilibria can also be characterized by the condition

$\xi_{\max } \equiv \frac{R}{a} \sqrt{4 \pi G \rho_{\mathrm{c}}}<6.451$,

where $R$ is the cloud's radius.

Recently, Lombardi \& Bertin (2001) have re-examined the classical Bonnor-Ebert problem relaxing the assumption of spherical shape of the cloud. The result is that for non-spherical isothermal clouds, the critical Bonnor-Ebert mass is larger than the value given by Eq. (1); a sphercal shape is more prone to gravitational instability than clouds of other shapes. The density contrast $\rho_{\mathrm{c}} / \rho_{\mathrm{b}}$ for marginally stable non-spherical clouds is different from the Bonnor-Ebert value 13.98 for spherical clouds, but, remarkably, the condition for stability, when expressed in terms of the ratio of the average density $\bar{\rho}$ to the boundary density $\rho_{\mathrm{b}}$, i.e. $\bar{\rho} / \rho_{\mathrm{b}}<2.465$, is independent of cloud shape.

To express the condition for stability $\left(M<M_{\mathrm{BE}}\right.$ and $\left.\rho_{\mathrm{c}} / \rho_{\mathrm{b}}<13.98\right)$ in terms of observable quantities one can eliminate the external pressure writing $p_{\mathrm{ext}}=a^{2} \rho_{\mathrm{b}}>13.98^{-1} a^{2} \rho_{\mathrm{c}}$, obtaining, for a given cloud's mass $M$,

$\rho_{\mathrm{c}}<19.53 \frac{a^{6}}{G^{3} M^{2}}$ 
that, for a cloud of molecular hydrogen with mean molecular weight $\mu=2.33$ becomes

$n_{\mathrm{c}}<1.6 \times 10^{5}\left(\frac{T_{\mathrm{g}}}{10 \mathrm{~K}}\right)^{3}\left(\frac{M}{M_{\odot}}\right)^{-2} \mathrm{~cm}^{-3}$.

Thus, stable isothermal equilibria are only possible provided the central density is relatively low (in the absence of magnetic fields): this causes a conflict with observations if one attempts to model observed molecular cores with such equilibrium configurations. For example, if dense cores with $M>4 M_{\odot}$ and $T_{\mathrm{g}}=10 \mathrm{~K}$ are modeled as stable Bonnor-Ebert spheres, their density according to Eq. (4) will fall below the critical value of $\sim 10^{4} \mathrm{~cm}^{-3}$ reqired to excite the molecular lines observed in these regions. Moreover, the central densities inferred in many pre-stellar cores based on the observed mm-submm dust emission (e.g. Ward-Thompson et al. 1999) is often above $10^{5} \mathrm{~cm}^{-3}$ and hence for gas temperatures of $\sim 10 \mathrm{~K}$, only cores of mass below $1 M_{\odot}$ can be in stable equilibrium.

Many of the observed objects however have higher masses (see the discussion of Bacmann et al. 2000) and also column densities of up to $10^{23} \mathrm{~cm}^{-2}$ in molecular hydrogen or 100 visual magnitudes of extinction. One can express the stability condition for Bonnor-Ebert spheres as a condition on the cloud's total (from edge to edge) extinction through the center $A_{\mathrm{V}, \text { tot }}=N\left(\mathrm{H}_{2}\right) / 10^{21} \mathrm{~cm}^{-2}$, obtaining

$A_{\mathrm{V}, \text { tot }} \lesssim 29\left(\frac{T_{\mathrm{g}}}{10 \mathrm{~K}}\right)^{2}\left(\frac{M}{M_{\odot}}\right)^{-1}$ mag.

This is clearly much less than often observed and provides a strong argument for the support of many observed structures being due to forces other than pure thermal pressure (though there are also cases of thermal support, e.g. Alves et al. 2001; Johnstone et al. 2000, 2001). In the following, we consider "observed cores" as having a minimum $A_{\mathrm{V}}$ of $10 \mathrm{mag}$, and thus exclude processes such as UV photoelectric effect from consideration. We do not moreover in this work compute models including magnetic fields and turbulence, but briefly mention in the next sections how these agent of support modify the picture outlined above.

In summary, non-magnetic stable isothermal cores represent only moderate enhancements (a factor $\sim 2.5$ in the average density) of the ambient gas density, quite independent on cloud shape and the turbulent field. This limited density range represents a serious limitation when modeling molecular cloud cores as non-magnetized hydrostatic equilibria. For example Boland \& de Jong (1984) were able to reproduce column density, brightness temperatures and abundances of several chemical species measured in dark clouds like L134, L183 and TMC-1 only with models characterized by density contrast $\rho_{\mathrm{c}} / \rho_{\mathrm{b}} \simeq 2000$. Hasegawa (1988) however verified that these models were well into the unstable regime. The same criticism was made by Chièze \& Pineau des Fôrets (1987) to the hydrostatic models computed by Falgarone \& Puget (1985). Hasegawa (1988) concluded that stable spherical, hydrostatic models able to satisfy all observational constraints on extinction, external pressure, mass, and chemical composition of molecular cloud cores, could no be constructed. In this respect, it is interesting to recall that when molecular cloud cores are fitted by Bonnor-Ebert spheres, the best fit is obtained for models that are in the unstable regime: $\xi_{\max }=6.9$ for B68 (Alves et al. 2001), $\xi_{\max }=12.5$ for B335 (Harvey et al. 2001, although B335 is not a starless core).

\subsection{Magnetic fields}

The additional support provided by a large-scale magnetic fields can be evaluated by a virial-theorem analysis or with the help of detailed calculations (see e.g. McKee et al. 1987, for a review). The maximum value of a cloud mass for the existence of stable equilibria is approximately equal to

$M_{\mathrm{cr}} \simeq\left[1-\left(\frac{0.13}{\lambda}\right)^{2}\right]^{-3 / 2} M_{\mathrm{BE}}$,

where $\lambda$ is the magnetic mass-to-flux ratio of the cloud in units of $G^{-1 / 2}$ (Mouschovias \& Spitzer 1976; Tomisaka et al. 1988). For magnetically subcritical clouds $(\lambda \lesssim 0.13)$, no amount of external compression can induce the collapse of the cloud (as long as the magnetic field remains frozen in the matter). Magnetically supercritical clouds $(\lambda \gtrsim 0.13)$ can exist in stable equilibrium for masses larger than the Bonnor-Ebert critical mass, and can reach values of $\rho_{\mathrm{c}} / \rho_{\mathrm{b}}$ much higher than 13.98 (up to $\sim 100$, see Figs. 4 and 6 of Tomisaka et al. 1988).

\subsection{Non-uniform turbulence and temperature gradients}

If the turbulent motions of the gas are characterized by a uniform (three-dimensional) mean square velocity $\left\langle\boldsymbol{v}_{\text {turb }}^{2}\right\rangle$, the effects of a turbulent pressure, in addition to the thermal pressure, can be trivially incorporated in the classic Bonnor-Ebert picture summarized above. The condition for the existence of stable equilibria is the same expressed by Eq. (1) with the sound speed $a^{2}$ replaced by an "effective" sound speed $a_{\mathrm{eff}}^{2}=a^{2}+\left\langle\boldsymbol{v}_{\mathrm{turb}}^{2}\right\rangle / 3$, allowing the existence of stable equilibria for values of the central density (or peak extinction) larger than for clouds supported by thermal pressure alone. Notice, however, that (as in the purely thermal case) marginally stable clouds are characterized by a density contrast $\rho_{\mathrm{c}} / \rho_{\mathrm{b}}=13.98$ or $\bar{\rho} / \rho_{\mathrm{b}}=2.465$, where $\bar{\rho}$ is the average density, irrespectively on the cloud's effective temperature.

One should remember also that the observed line widths (which in some cases are close to being thermal) place limits on possible values of $\boldsymbol{v}_{\mathrm{turb}}$. In dense cores, as well as in giant molecular clouds, turbulent linewidths are often observed to increase at larger scales (see discussion in Barranco \& Goodman 1998; Goodman et al. 1998), suggesting that these objects can be modeled as negative-index polytropes (de Jong et al. 1980; Dickman \& Clemens 1983; Maloney 1988). This is easily understood comparing the polytropic equation of state $P \propto \rho^{1+1 / n}$ with the expression for the thermal pressure of a gas, $P \propto \rho T$, implying $T \propto \rho^{1 / n}$. Since the density is always a decreasing function of radius, negative index polytropes represent equilibrium configurations in which the effective temperature increases outward. However, from the point of view of the 
stability properties, our models show a behavior similar to polytropes with $n \gg 1$. We will return to this point in Sect. 6.2.

The logatropic equation of state analyzed by McLaughlin \& Pudritz (1996) to incorporate the contribution of non-thermal motions to the support of molecular clouds (and cloud cores) makes possible the existence of stable equilibria for large density constrasts $\left(\rho_{\mathrm{c}} / \rho_{\mathrm{b}} \simeq 100\right)$ but the resulting configurations are much more extended than Bonnor-Ebert spheres and are characterized by a maximum ratio $\bar{\rho} / \rho_{\mathrm{b}}=3 / 2$, even smaller than for critical Bonnor-Ebert spheres. Indeed, the observed column density distributions (Bacmann et al. 2000) of cores do not fit well to logatropes.

\section{Model assumptions}

Following the approach outlined by Goldsmith (2001), we compute the gas thermal equilibrium assuming that the dust temperature is unaffected by collisions with gas particles (see Evans et al. 2001, for estimates of the validity of this). Moreover, we have used the analytical fit of Zucconi et al. (2001) to determine dust temperature as a function of radius in our spherically symmetric model cores. The justification for this is simplicity. We are interested here in understanding qualitatively to what extent departures from isothermality may affect the properties of pre-stellar cores. High precision in determining the gas cooling rates and the dust temperature distribution is unlikely to fundamentally affect our results.

\subsection{Dust and gas temperature}

Thus we determine the gas temperature $T_{\mathrm{g}}$ by solving the equation of thermal balance of the gas in the presence of dust grains heated by the external radiation field,

$\Gamma_{\mathrm{cr}}-\Lambda_{\mathrm{g}}-\Lambda_{\mathrm{gd}}=0$

where $\Gamma_{\mathrm{cr}}$ is the cosmic-ray heating rate, $\Lambda_{\mathrm{g}}$ the gas cooling rate by molecular and atomic transitions, and $\Lambda_{\mathrm{gd}}$ the gas-dust energy transfer rate. We follow Goldsmith (2001) and adopt a cosmic-ray heating rate

$\Gamma_{\mathrm{cr}}=10^{-27}\left(\frac{\zeta}{3 \times 10^{-17} \mathrm{~s}^{-1}}\right)\left[\frac{n\left(\mathrm{H}_{2}\right)}{\mathrm{cm}^{-3}}\right] \mathrm{erg} \mathrm{cm}^{-3} \mathrm{~s}^{-1}$.

The gas cooling rate $\Lambda_{\mathrm{g}}$ for typical conditions of molecular cloud cores has been computed by Goldsmith (2001) in the LVG approximation (assuming a velocity gradient of $1 \mathrm{~km} \mathrm{~s}^{-1} \mathrm{pc}^{-1}$ ) for various degrees of depletion of molecules from the gas phase onto grain surfaces. We adopt the parametrization of Goldsmith (2001)

$\Lambda_{\mathrm{g}}=\alpha\left(\frac{T_{\mathrm{g}}}{10 \mathrm{~K}}\right)^{\beta} \operatorname{erg~cm}^{-3} \mathrm{~s}^{-1}$,

where $\alpha$ and $\beta$ are parameters that depend on the $\mathrm{H}_{2}$ density and the molecular depletion factors. When not explicitly noted, we assume that molecular abundances in the gas have their standard (undepleted) values. As for the the gas-dust energy transfer rate we adopt the expression given by Goldsmith (2001) based on calculations of Burke \& Hollenbach (1983),

$$
\begin{aligned}
\Lambda_{\mathrm{gd}}= & 2 \times 10^{-33}\left(\frac{n}{\mathrm{~cm}^{-3}}\right)^{2}\left(\frac{T_{\mathrm{g}}-T_{\mathrm{d}}}{\mathrm{K}}\right) \\
& \times\left(\frac{T_{\mathrm{g}}}{10 \mathrm{~K}}\right)^{1 / 2} \operatorname{erg~cm}{ }^{-3} \mathrm{~s}^{-1} .
\end{aligned}
$$

To determine the dust temperature $T_{\mathrm{d}}$ we ignore the gas-dust coupling and we solve the equation of thermal balance of the dust

$\Gamma_{\mathrm{ext}}-\Lambda_{\mathrm{d}}=0$

where $\Gamma_{\text {ext }}$ is the dust heating rate by the external radiation field and $\Lambda_{d}$ the dust cooling rate by radiation. In practice, we use the analytical solution of Zucconi et al. (2001) to compute $T_{\mathrm{d}}$ as function of extinction from the cloud's boundary.

As in Zucconi et al. (2001), we have adopted as a standard the interstellar radiation field (ISRF) in the solar neighborhood given by Mathis et al. (1983) and Black (1994). It consists of four components: the V-NIR component, peaking at $\lambda \simeq 1 \mu \mathrm{m}$, the MIR and FIR components, peaking at $\lambda \simeq 100$ and $140 \mu \mathrm{m}$ respectively, and the cosmic background radiation, peaking at $\lambda \simeq 1 \mathrm{~mm}$. We allow for variations of the ISRF, assuming, for simplicity, that the spectral shapes of these components are the same as the local ISRF, and we scale the intensity of the V-NIR, MIR, and FIR components by a factor $G_{0}$ (with $G_{0}=1$ for the local ISRF).

The existence of a cloud radius leads to the necessity of an external pressure to keep the system in equilibrium. We embed our model cloud cores in a spherical envelope that represents the ambient molecular cloud. We do not need to specify the physical properties of this envelope; we just assume that it provides the needed external pressure and a shielding from the ISRF directly incident on the cloud cores. We set the latter at the value $A_{\mathrm{V}}^{\text {env }}=1$ (McKee 1999), sufficient to absorb the UV and most of the V-NIR radiation. We define $A_{\mathrm{V}, \mathrm{c}}$ to be the centre-to-edge extinction of our model cloud cores. The range of masses and densities of interest here corresponds to the range $5 \lessgtr A_{\mathrm{V}, \mathrm{c}} \lesssim 100$, where the central dust temperature is determined mostly by optically thick absorption of V-NIR radiation and optically thin absorbtion of MIR and FIR radiation.

The fundamental parameters of our models are the external pressure $p_{\text {ext }}$ and the intensity of the external radiation field $G_{0}$. In this paper, we consider independent variations of these two quantities, and we analyse separately the dependence of the critical mass for increasing $p_{\text {ext }}$ and fixed $G_{0}$ (Sect. 5, Fig. 2), and the effect of varying $G_{0}$ for a given value of $p_{\text {ext }}$ (Sect. 6, Fig. 6).

\subsection{Effects of molecular depletion}

We include in our models the effect of molecular depletion onto dust grains, parametrized for simplicity by the single parameter $f_{\mathrm{d}}$ (depletion factor), defined as the ratio of the "standard" abundance of $\mathrm{CO}$ isotopes and CS to the actual abundances. The corresponding abundances of other molecular species are given in Table 3 of Goldsmith (2001). Gas-phase cooling rates for several values of $f_{\mathrm{d}}$ in the range 1-100 were computed and 
tabulated by Goldsmith (2001) for $\mathrm{H}_{2}$ density from $10^{3} \mathrm{~cm}^{-3}$ to $10^{6} \mathrm{~cm}^{-3}$. We assume the dependence of $f_{\mathrm{d}}$ on density given by Tafalla et al. (2002),

$f_{\mathrm{d}}=\exp \left(\frac{n}{n_{\mathrm{dep}}}\right)$

where the critical density for $\mathrm{CO}$ depletion is taken to be $n_{\text {dep }}=$ $5.5 \times 10^{4} \mathrm{~cm}^{-3}$.

Although we are mainly interested in applications to molecular cloud cores of moderate to high extinction, where molecular depletion is effective only in a region of limited spatial extent, one should keep in mind that large fraction of the cloud's mass is contained in the outer parts (roughly $M(r) \propto r$ ), and therefore the overall stability of the cloud may be modified by the thermal properties of these external layers.

In our models we find in general that the effects of depletion on the temperature of the gas are significant only for densities $n \lesssim 10^{5} \mathrm{~cm}^{-3}$. At higher density, gas-dust coupling overwhelms the reduction of the cooling rate of the gas, and the gas temperature becomes insensitive to depletion. This is in qualitative agreement with the results of Goldsmith (2001). We conclude with Goldsmith (2001) that molecular depletion in the central, dense regions of molecular cloud cores $(n \gtrsim$ $10^{4.5}-10^{5} \mathrm{~cm}^{-3}$ ) is not associated with an increase in the gas temperature.

In the extreme case where $\mathrm{CO}$ (and other molecules containing $\mathrm{C}, \mathrm{N}, \mathrm{O}, \mathrm{S}$, etc.) depletes out onto grains causing Eq. (7) to reduce to $\Gamma_{\mathrm{cr}}=\Lambda_{\mathrm{gd}}$. Then using Eqs. (8) and (10), one finds

$\frac{T_{\mathrm{g}}-T_{\mathrm{d}}}{T_{\mathrm{g}}} \simeq 5\left(\frac{\zeta}{3 \times 10^{-17} \mathrm{~s}^{-1}}\right)\left(\frac{n}{10^{4} \mathrm{~cm}^{-3}}\right)^{-1}\left(\frac{T_{\mathrm{g}}}{10 \mathrm{~K}}\right)^{-1.5}$.

This shows that in the extreme case where all heavy species deplete out, coupling between gas and dust temperatures will be assured for densities larger than $5 \times 10^{4} \mathrm{~cm}^{-3}$ if the cosmic ray ionization rate is standard or lower. Since it is essentially at densities above this value that $\mathrm{CO}$ is observed to deplete out, we conclude that a rough equality between gas and dust temperatures in the depleted region is a reasonable assumption. In this context, it is interesting to note that Bergin et al. (2002) suggest that even such relatively volatile species as molecular nitrogen have depleted out in the central core of B68.

\section{Temperature distributions for L1544 and B68}

Before considering what one expects for theoretical equilibrium cores, it is useful to consider what one predicts for the temperature variation within an object whose density structure is similar to those observed. Specifically, we will consider the case of L1544 (Caselli et al. 2002a, 2002b) and B68 Alves et al. (2001), two well studied prestellar cores.

To model L1544 we assume $G_{0}=1$ and the density structure of Tafalla et al. (2002), which, in turn, is based on the $1.3 \mathrm{~mm}$ dust emission and absorption maps of Bacmann et al. (2000). Thus, we assume the $\mathrm{H}_{2}$ density distribution $n(r)$ to be given by

$n(r)=\frac{n_{\mathrm{c}}}{1+\left(r / r_{0}\right)^{2.5}}$, where the central $\mathrm{H}_{2}$ density is $n_{\mathrm{c}}=1.4 \times 10^{6} \mathrm{~cm}^{-3}$ and $r_{0}=0.014 \mathrm{pc}$. It is worth noting that we have on purpose taken here a somewhat extreme case with large density contrast relative to the surroundings and large central column density $\left(6.0 \times 10^{22} \mathrm{~cm}^{-2}\right.$ corresponding to $60 \mathrm{mag}$ of extinction, from Bacmann et al. 2000). One should also be aware that the aspect ratio of the core based on the dust emission contours is $\sim 0.6$ and hence spherical symmetry is a crude assumption. Nevertheless, the case is of interest in that it is a model which approximates the actually observed density distribution and in which, one expects that the central temperature will be relatively low (due to the large extinction).

For B68 we assume the density structure of a BonnorEbert sphere with the physical parameters given by Alves et al. (2001). The central density $n_{\mathrm{c}}=2.4 \times 10^{5} \mathrm{~cm}^{-3}$ is a factor $\sim 6$ lower than in the case of L1554, and consequently the amount of $\mathrm{CO}$ depletion at the center of B68 is about two orders of magnitude lower than at the center of L1544. The intensity of the IRSF incident on B68 can be estimated as follows. We first estimate the external UV radiation field based on the $90 \mu \mathrm{m}$ data of Ward-Thompson et al. (2002) and the conversion between FIR intensity and incident field found by Boulanger \& Perault (1988). Another approach is to use the $7 \mu \mathrm{m}$ intensity from Bacmann et al. (2000) and the results of Boulanger et al. (1996) to infer $G_{0}$. Combining these, we conclude that the radiation field incident on B68 is 2.5 times larger that the standard ISRF.

In Fig. 1, we show the inferred gas temperature dependence for L1544 and B68, computed with cosmic ray ionization rate $\zeta=1.3 \times 10^{-17} \mathrm{~s}^{-1}$ and accomodation coefficient $=0.3$. The assumed CO depletion factor (cut off at a value of 100) is shown for comparison. In the inner part of both cores (densities above $3 \times 10^{4} \mathrm{~cm}^{-3}$ ), the gas temperature is coupled to that of the dust and thus decreases gradually towards the core center. This coupling is more effective in the case of L1544, which has a higher central density. The gas is slightly hotter than the dust however due to cosmic ray heating and this difference increases as the density decreases. The $\mathrm{CO}$ depletion appears to have only small effects on the gas temperature profile because while at high density, gas-dust coupling dominates, at densities below $10^{5} \mathrm{~cm}^{-3}$, the depletion is not very large (and the optically thick nature of $\mathrm{CO}$ cooling causes the dependence of CO cooling on abundance to be minor). We draw the conclusion from this study that in the depleted core nucleus (densities above $10^{5} \mathrm{~cm}^{-3}$ ) where many molecular species may be unobservable because they are in solid form, the gas temperature may be expected to be close to that of the dust. In outer regions where the density is below $10^{5} \mathrm{~cm}^{-3}$ and where shielding from the external radiation field is much less, the gas and dust temperatures become uncoupled and one may find gas temperatures lower than that of the dust.

It is worth pointing out that the scenario depicted in Fig. 1 yields gas temperatures in conflict with observation. Tafalla et al. (2002) find based on their $\mathrm{NH}_{3}$ observations a temperature of $8.7 \mathrm{~K}$ throughout the L1544 core with no evidence for temperature gradients ( $40^{\prime \prime}$ HPBW equivalent to 0.03 parsec), whereas Bourke et al. (1995) give $T_{\mathrm{g}}=16 \mathrm{~K}$ for B68, also derived from $\mathrm{NH}_{3}$ data. These are in rough agreement with the 


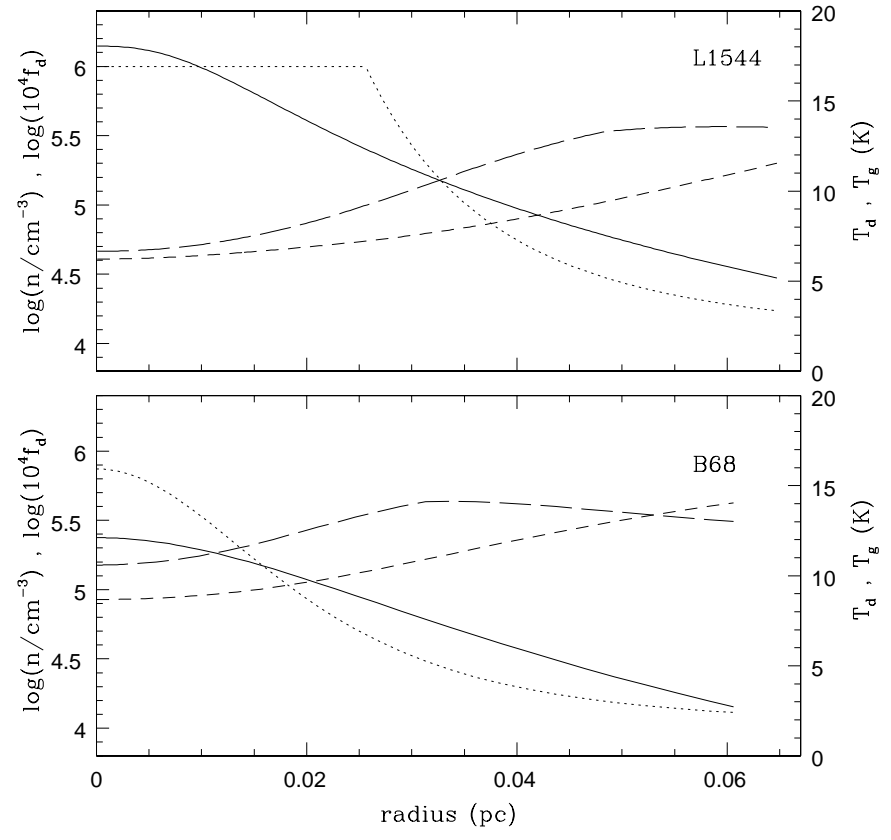

Fig. 1. Upper panel: inferred gas (long-dashed curve) and dust temperature dependence (short-dashed curve) for the model of L1544 discussed in the text (with $G_{0}=1$ ). The assumed density distribution from Eq. (14) is shown for comparison (solid curve). The assumed CO depletion factor $f_{\mathrm{d}}$ (multiplied by $10^{4}$, dotted curve) is also shown. Lower panel: same as above, for the model of B68 (with $G_{0}=2.5$ ). One sees that for $n>3 \times 10^{4} \mathrm{~cm}^{-3}$ the gas temperature follows the dust temperature and decreases towards the core center. Outside this region, gas and dust are decoupled and the gas heating is determined solely by cosmic ray ionization.

values in Fig. 1 though the L1544 data do not show evidence for increasing temperature with radius as predicted.

Another conclusion that one can draw from Fig. 1 is that the gas temperature and hence pressure in the high density core nucleus depends somewhat on the external radiation field even for cores of extremely high visual extinction. Thus one can expect cores in Taurus for example (where the incident radiation field appears to be average) to have different characteristics than Ophiuchus cores where the radiation field appears in general to be an order of magnitude higher. Of course, there are other effects such as the external pressure which may differ in the two cases also but we stress that the radiation field alone may cause core temperatures and hence accretion rates to be higher in Ophiuchus than in Taurus.

\section{Self consistent hydrostatic equilibrium results}

The equation of hydrostatic equilibrium for a spherically symmetric cloud is

$\frac{\mathrm{d} p}{\mathrm{~d} r}+\rho \frac{\mathrm{d} V}{\mathrm{~d} r}=0$

where $p$ is the gas pressure and $V$ the gravitational potential. The latter satisfies Poisson's equation, which in spherical symmetry reads

$\frac{1}{r^{2}} \frac{\mathrm{d}}{\mathrm{d} r}\left(r^{2} \frac{\mathrm{d} V}{\mathrm{~d} r}\right)=4 \pi G \rho$

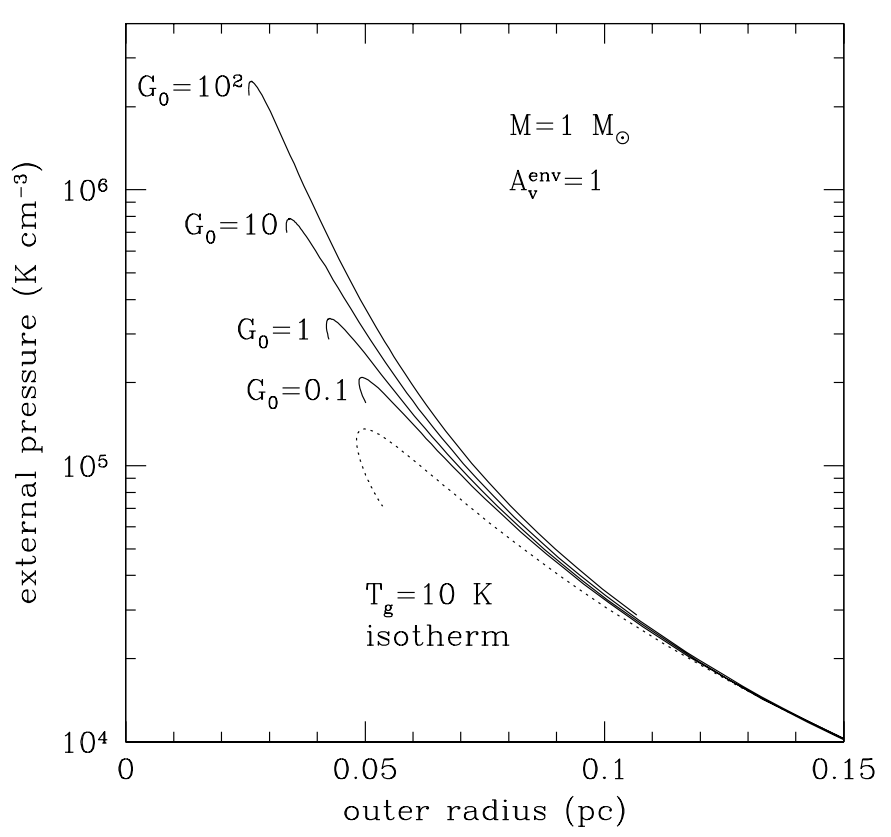

Fig. 2. Sequences of model clouds with $M=1 M_{\odot}$ and $A_{\mathrm{V}}^{\text {env }}=1$ in the radius-pressure diagram, for various scaling factors $G_{0}$ of the ISRF (solid lines). For comparison, the dotted line shows a Bonnor-Ebert isotherm with $T_{\mathrm{g}}=10 \mathrm{~K}$ for the same cloud mass.

The gas pressure $p$, neglecting the contribution of turbulent pressure, is

$p=\frac{\rho}{\mu m_{\mathrm{H}}} k T_{\mathrm{g}}$,

where $T_{\mathrm{g}}$ is the gas temperature, computed as described in Sect. 3, and $\mu=2.33$ the mean molecular weight.

The behavior of our model clouds with respect to an external compression is qualitatively similar to that of isothermal spheres. Figure 2 shows the locus in the radius-external pressure diagram of cloud models with $M=1 M_{\odot}$, for different values of the ISRF intensity $G_{0}$. For large radii the cloud follows closely the $T_{\mathrm{g}}=10 \mathrm{~K}$ Bonnor-Ebert isotherm, independent of the radiation field (the effect of gas-dust coupling is negligible for these diffuse configurations). At higher values of the external pressure the cloud radius is smaller and the central (and average) density becomes larger. As a result, the central gas temperature increases and the sequence of equilibria departs from the Bonnor-Ebert isotherm. Eventually, a critical value of the pressure is reached above which no equilibria exist, and the curve turns down. Past this critical point, equilibria are characterized by an increasing central density and density contrast, and are unstable (see following section). For clarity, in Fig. 2 we truncate the curves of equilibrium slightly after the critical point.

Figure 3 shows the density of $\mathrm{H}_{2}$, the temperature of the gas and the temperature of the dust as function of radius for the marginally stable clouds of mass $M=1 M_{\odot}$ with $G_{0}=1$ (central $\mathrm{H}_{2}$ density $n_{\mathrm{c}}=2.7 \times 10^{5} \mathrm{~cm}^{-3}$ ). The density profile is very close to that of an isothermal sphere with the same values of the central density, shown for comparison in Fig. 3 for temperatures equal to the central and boundary temperature of the model. The gas temperature decreases from the outer 


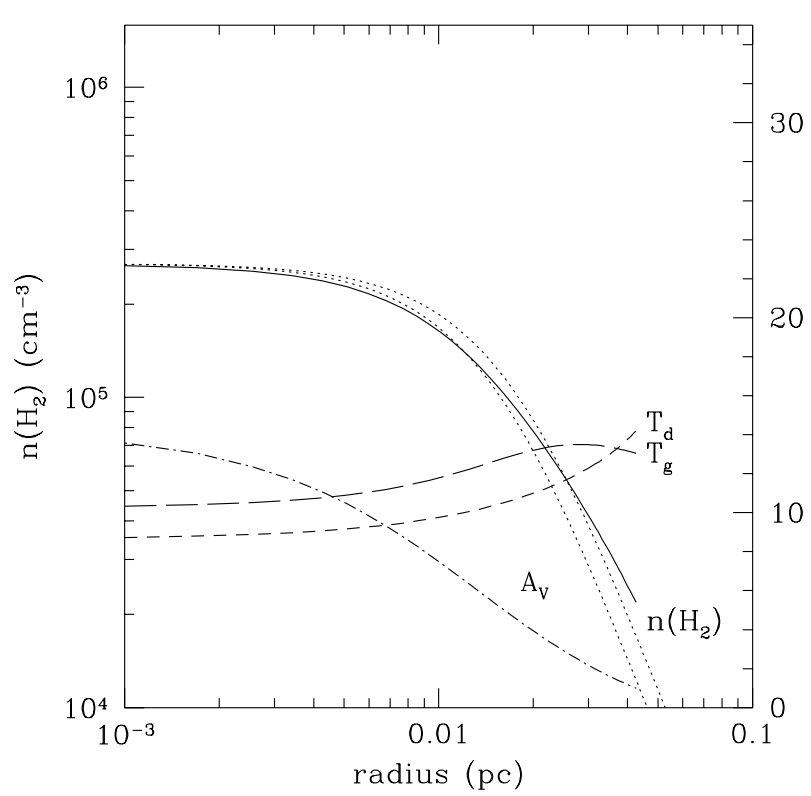

Fig. 3. Density of $\mathrm{H}_{2}$ (solid line), temperature of the dust (shortdashed line), temperature of the gas (long-dashed line), and visual extinction (dash-dotted line) as function of radius for the marginally stable clouds with $M=1 M_{\odot}$ and $G_{0}=1$. The dotted lines show for comparison the density profile of an isothermal sphere with the same values of $n_{\mathrm{c}}$ and $T=T_{\mathrm{g}, \mathrm{c}}$ or $T_{\mathrm{g}, \mathrm{b}}$. The visual extinction at the cloud's center is $A_{\mathrm{V}, \mathrm{c}}=14$.

Table 1. Properties of marginally stable clouds with $M=1 M_{\odot}$.

\begin{tabular}{llllll}
\hline \hline$G_{0}$ & $\begin{array}{l}n_{\mathrm{c}} \\
\left(\mathrm{cm}^{-3}\right)\end{array}$ & $\rho_{\mathrm{c}} / \rho_{\mathrm{b}}$ & $\begin{array}{l}T_{\mathrm{g}, \mathrm{c}} \\
(\mathrm{K})\end{array}$ & $\begin{array}{l}T_{\mathrm{d}, \mathrm{c}} \\
(\mathrm{K})\end{array}$ & $A_{\mathrm{V}, \mathrm{c}}$ \\
\hline 0.1 & $1.5 \times 10^{5}$ & 10.0 & 9.2 & 6.2 & 9 \\
1 & $2.7 \times 10^{5}$ & 12.0 & 8.7 & 10.3 & 14 \\
10 & $5.8 \times 10^{5}$ & 14.4 & 12.7 & 12.0 & 22 \\
$10^{2}$ & $1.3 \times 10^{6}$ & 15.1 & 17.0 & 16.8 & 38 \\
\hline
\end{tabular}

regions to the center of the cloud. For marginally stable clouds with $M=1 M_{\odot}$ the central density is sufficient to ensure a modest gas-dust coupling, but the gas temperature is mainly determined by the balance of cosmic-ray heating and gas cooling.

Figure 4 shows the $\mathrm{H}_{2}$ density, the gas and dust temperatures and the extinction profile of the marginally stable cloud in thermal equilibrium with an ISRF with $G_{0}=100$ and bounded by an external pressure $p_{\text {ext }}=1 \times 10^{6} \mathrm{~K} \mathrm{~cm}^{-3}$. These roughly correspond to the structures observed by Johnstone et al. (2001) in the $\rho$ Oph and Orion clouds. For comparison, the figure also shows the density profiles of marginally stable Bonnor-Ebert spheres with the same central density and uniform temperature equal to the gas temperature at the center and at the boundary of the cloud.

In Tables 1 and 2 we list the values of central $\mathrm{H}_{2}$ density, gas and dust temperature, density contrast, and optical extinction at the center for marginally stable clouds with $M=1$ and $5 M_{\odot}$ and various scaling factors for the ISRF. In these models we have varied $p_{\text {ext }}$ until we find the marginally stable model.

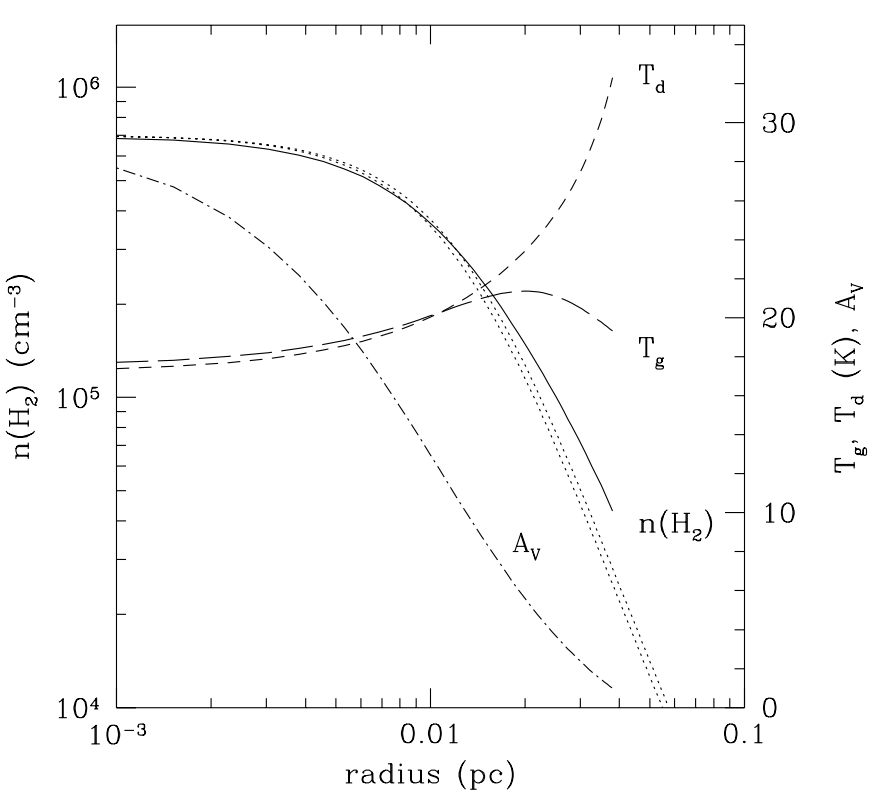

Fig. 4. Same as in Fig. 3, but for a marginally stable cloud bounded by an external pressure $p_{\text {ext }}=1 \times 10^{6} \mathrm{~K} \mathrm{~cm}^{-3}$ and with $G_{0}=100$. The mass of the cloud is $M=1.6 M_{\odot}$ and the extinction from the center to the edge of the cloud is $A_{\mathrm{V}}=29$.

Table 2. Properties of marginally stable clouds with $M=5 M_{\odot}$.

\begin{tabular}{llllll}
\hline \hline$G_{0}$ & $\begin{array}{l}n_{\mathrm{c}} \\
\left(\mathrm{cm}^{-3}\right)\end{array}$ & $\rho_{\mathrm{c}} / \rho_{\mathrm{b}}$ & $\begin{array}{l}T_{\mathrm{g}, \mathrm{c}} \\
(\mathrm{K})\end{array}$ & $\begin{array}{l}T_{\mathrm{d}, \mathrm{c}} \\
(\mathrm{K})\end{array}$ & $A_{\mathrm{V}, \mathrm{c}}$ \\
\hline 0.1 & $9.9 \times 10^{3}$ & 19.2 & 10.0 & 9.4 & 3 \\
1 & $1.1 \times 10^{4}$ & 20.0 & 10.0 & 14.2 & 3 \\
10 & $1.5 \times 10^{4}$ & 24.2 & 10.0 & 21.4 & 4 \\
$10^{2}$ & $2.4 \times 10^{4}$ & 34.4 & 10.5 & 32.3 & 5 \\
\hline
\end{tabular}

A comparison of the results for the two different masses is instructive. Small mass clouds $\left(M \simeq 1 M_{\odot}\right)$ exposed to the average ISRF behave like isothermal spheres with $T_{\mathrm{g}} \simeq 10 \mathrm{~K}$, and are characterized by a maximum density contrast and critical central density very close to those of the corresponding Bonnor-Ebert values. Increasing the ISRF $\left(G_{0}=10\right)$ has the effect of raising the dust temperature, and of increasing slightly the gas temperature in the central regions. This increase in the central gas temperature causes a higher central density in the marginally stable core and this, in turn, increases the efficiency of dust-gas coupling. A further increase of the ISRF $\left(G_{0}=100\right)$ establishes the approximate equality of the gas and dust temperatures at the center. Thus, the fact that small mass clouds are dynamically stable for larger values of the central density than their more massive counterparts (see Eq. (4)), has the consequence that they are "stabilized" by an external ISRF simply because they are able to become warmer if the dust does. Their gas temperature profiles, however, remain quite uniform, and therefore the maximum density contrast cannot deviate significantly from the Bonnor-Ebert value $\rho_{\mathrm{c}} / \rho_{\mathrm{b}} \simeq 13.98$, as shown in Table 1.

A different behaviour characterizes more massive clouds $\left(M \simeq 5 M_{\odot}\right)$, as they become dynamically unstable before an efficient gas-dust coupling can be established, for any value of 


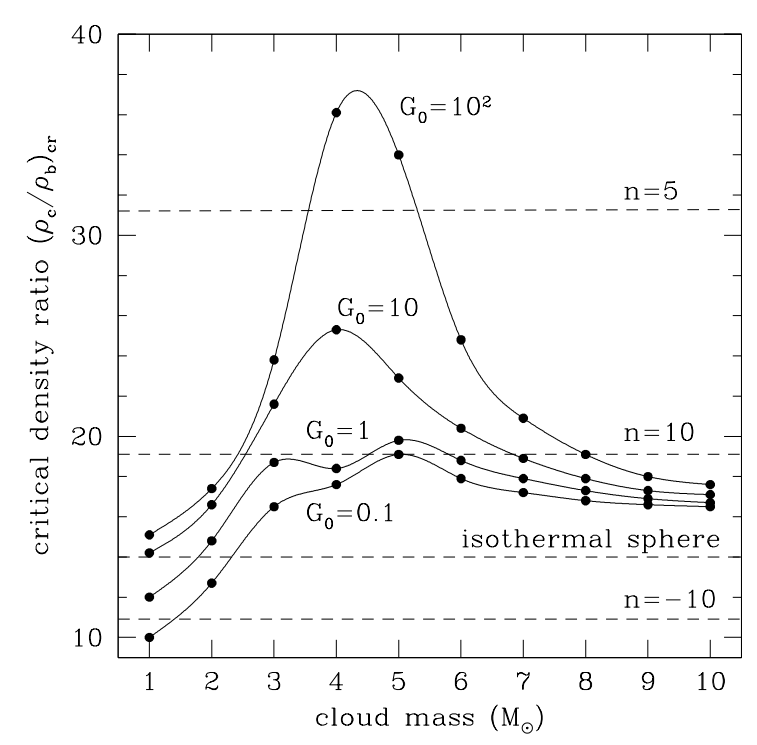

Fig. 5. Value of the central-to-boundary density ratio $\left(\rho_{\mathrm{c}} / \rho_{\mathrm{b}}\right)_{\mathrm{cr}}$ as function of the cloud's mass for different scaling factors $G_{0}$ of the ISRF (dots and solid lines). The dashed lines show the corresponding values of $\left(\rho_{\mathrm{c}} / \rho_{\mathrm{b}}\right)_{\mathrm{cr}}=13.98$ for marginally stable isothermal and polytropic spheres of index $n$.

the intensity of the ISRF (see Table 2). Their central extinction is low $\left(A_{\mathrm{V}, \mathrm{c}} \simeq 3-5\right)$, and hence they do not correspond to "real observed cores". Nevertheless, it is interesting that they can develop significant inward temperature gradients, because molecular cooling is less efficient than cosmic-ray heating in the central regions, characterized by densities $n \simeq 10^{4}-10^{5} \mathrm{~cm}^{-3}$ (where $\Lambda_{\mathrm{g}} \propto n^{0.5}$ ) than in the outer parts where $n \simeq 10^{3} \mathrm{~cm}^{-3}$ (and $\Lambda_{\mathrm{g}} \propto n$, as cosmic-ray heating). An inward temperature gradient stabilizes the cloud (see Sect. 6), and allows large deviations in the maximum density contrast $\rho_{\mathrm{c}} / \rho_{\mathrm{b}}$, up to 30-40, as shown in Table 2 . Notice that any molecular depletion effect, ignored in these calculation, would make the central temperature even higher, and thus increase the slope of the temperature gradient.

In Fig. 5 we summarize our results for the maximum density contrast $\rho_{\mathrm{c}} / \rho_{\mathrm{b}}$ allowed for clouds in the mass range $M=1-$ $10 M_{\odot}$. Again $p_{\text {ext }}$ has been varied until the marginally stable model is found. Deviations of $\rho_{\mathrm{c}} / \rho_{\mathrm{b}}$ from the Bonnor-Ebert value 13.98 indicate deviations from a condition of uniform gas temperature in the cloud. As anticipated in the above discussion, the largest deviations from isothermality are obtained for masses $M \simeq 4-6 M_{\odot}$. Marginally stable clouds of small mass $\left(M \lesssim 2 M_{\odot}\right)$ are characterized by relatively large values of the central density, and by a sufficiently good coupling of gas and dust: they are uniformly warmer or colder according to the intensity of the external ISRF, but because of this coupling they do not develop significant temperature gradients, and therefore behave essentially as isothermal spheres. Marginally stable high-mass clouds $\left(M \gtrsim 8 M_{\odot}\right)$ also are similar to isothermal spheres, but for a different reason: they are characterized by relatively low values of the central density $\left(n_{\mathrm{c}} \simeq 10^{3} \mathrm{~cm}^{-3}\right)$ and also have low $A_{\mathrm{V}, \mathrm{c}}$ (unlike observed cores), which implies an uniform value of the gas temperature since both cosmic-ray heating and radiative gas cooling depend approximately linearly on density (and gas-dust coupling plays no role at these densities). In the intermediate mass range, both because of the different dependence of heating and cooling rates from density and partial gas-dust coupling, the deviations from uniform gas temperature are larger, and the maximum density contrast $\rho_{\mathrm{c}} / \rho_{\mathrm{b}}$ has a peak for $M \simeq 4-5 M_{\odot}$. Figure 5 also shows, for comparison, the values of the density contrast for marginally stable polytropic spheres of index $n$, discussed in the next section.

\section{The effect of the external radiation field}

The presence of an external ISRF affects the stability of clouds bounded by an external pressure. In the first place, it makes the dust (and therefore the gas) warmer on average, thus increasing the critical mass for gravitational instability (proportional to $T_{\mathrm{g}}^{2}$ ); in the second place, it enforces a temperature gradient in the cloud which modifies the value of the classic BonnorEbert critical mass.

The latter point is best investigated by the so-called static method originally devised by Zeldovich (1963) for white dwarfs and neutron stars (see Tassoul 1978). This method allows one to perform a stability analysis for axisymmetric motions on the basis of the properties of the equilibrium models only. The static criterion asserts that a turnover in the $M\left(\rho_{\mathrm{c}}\right)$ curves at fixed $P_{\text {ext }}$, occurring at say $\rho_{\mathrm{c}}=\rho_{\mathrm{c}}^{\mathrm{cr}}\left(p_{\text {ext }}\right)$, marks the onset of dynamical instability to radial motions. The condition $\left(\partial M / \partial \rho_{\mathrm{c}}\right)_{p_{\mathrm{ext}}}=0$ is both a sufficient and necessary condition for a stability transition: equilibria with $\rho_{\mathrm{c}}<\rho_{\mathrm{c}}^{\mathrm{cr}}\left(p_{\mathrm{ext}}\right)$ are stable, while equilibria with $\rho_{\mathrm{c}}>\rho_{\mathrm{c}}^{\mathrm{cr}}\left(p_{\text {ext }}\right)$ are unstable to at least one normal mode.

In Fig. 6 we show how the intensity of the external ISRF may affect the stability of a cloud bounded by a given external pressure. For this particular example, we have chosen $p_{\text {ext }}=2 \times 10^{4} \mathrm{~K} \mathrm{~cm}^{-3}$ (McKee 1999). The three curves labelled with $G_{0}=1,10$ and 100 show the mass versus central $\mathrm{H}_{2}$ density for externally heated clouds, compared with the similar quantities for isothermal spheres. According to the static method, continuous (dashed) lines represent stable (unstable) equilibria. The dotted lines indicates the boundaries of the stability domain for isothermal spheres with arbitrary temperatures (only the case $T_{\text {gas }}=10 \mathrm{~K}$ is shown) and non-isothermal spheres in thermal equilibrium with the external ISRF. The figure shows that for a given external pressure the condition of thermal equilibrium allows the existence of stable cloud configurations for values of mass and central density not permitted under the condition of uniform gas temperature.

Although not very large, this effect may resolve in part the problem posed by objects like B68 which are well fitted by slightly unstable Bonnor-Ebert spheres (magnetic fields are of course another possibility). For example, for the three marginally stable non-isothermal clouds of Fig. 6 the centralto-boundary density ratio are $\left(\rho_{\mathrm{c}} / \rho_{\mathrm{b}}\right)=19,25$ and 39 , for $G_{0}=1,10$ and 100 , compared with the corresponding ratio 13.98 for isothermal spheres, and 16.5 inferred for B68 (Alves et al. 2001). 


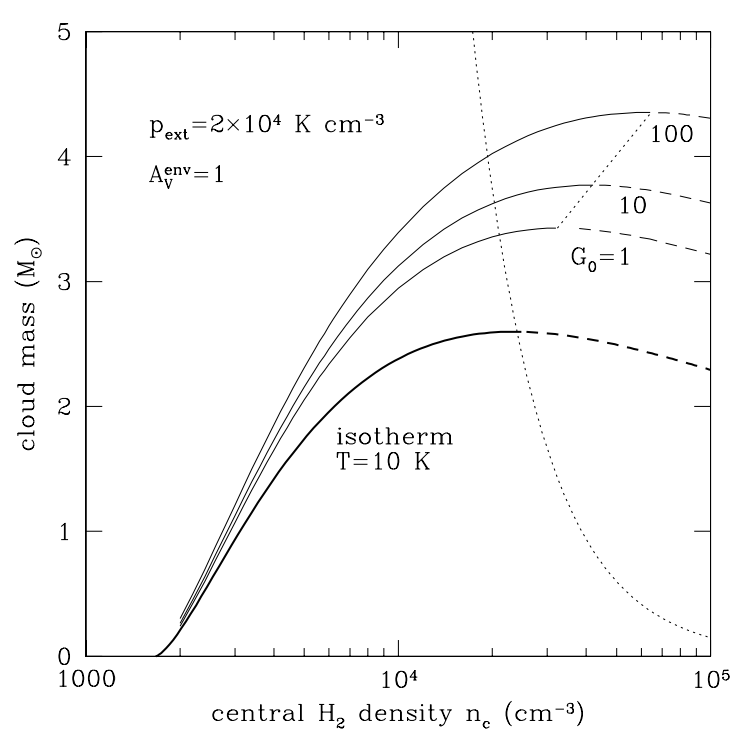

Fig. 6. Instability in model cloud cores for isothermal and nonisothermal models. The cloud's mass is plotted as function of the central $\mathrm{H}_{2}$ density $n_{\mathrm{c}}$ for $p_{\mathrm{ext}}=2 \times 10^{4} \mathrm{~K} \mathrm{~cm}^{-3}$ (solid curves, stable equilibria; dashed curves, unstable equilibria). Instability sets in at the maximum of $M\left(n_{\mathrm{c}}\right)$. The curves are labelled with the values of $G_{0}$. The dotted lines separate the regions of stability and instability for this value of $p_{\text {ext }}$ and arbitrary values of the temperature (in the isothermal case) and $G_{0}$ (in the non-isothermal case). The thick curve show the mass versus central density relation for an isothermal sphere with $T_{\mathrm{g}}=10 \mathrm{~K}$.

\subsection{Comparison with polytropic models}

From the point of view of the stability properties and the maximum density contrast, the models shown in this paper have a behavior similar to polytropes with $n \gg 1$ (see Fig. 5). Increasing the intensity of the ISRF produces an increase in the dust temperature which is felt by the gas only in the central parts of the cloud, where the coupling of dust and gas is more efficient. As a result, the central gas temperature $T_{\mathrm{g}, \mathrm{c}}$ increases slightly, whereas the gas temperature at the boundary $T_{\mathrm{g}, \mathrm{b}}$ remains unchanged $\left(T_{\mathrm{g}, \mathrm{b}} \simeq 10 \mathrm{~K}\right)$, since for densities $n \simeq 10^{3} \mathrm{~cm}^{-3}$ both the gas cooling rate and the the cosmic-ray heating rate are roughly proportional to $n$. Thus, one can extend Eq. (1) to polytropic clouds bounded by the same external pressure and characterized by the same thermal sound speed $a_{\mathrm{b}}$ at the boundary, and write

$M_{\mathrm{cr}} \simeq \alpha_{n} \frac{a_{\mathrm{b}}^{4}}{\sqrt{G^{3} p_{\mathrm{ext}}}}$.

Table 3 shows the value of $\alpha_{n}$ and the density contrast $\rho_{\mathrm{c}} / \rho_{\mathrm{b}}$ for clouds with different polytropic index $n$. For decreasing positive $n$, the coefficient $\alpha_{n}$ increases, indicating a larger critical mass than an isothermal sphere bounded by the same external pressure and with the same temperature at the boundary. For $n=6$, for example, the critical mass is expected to be a factor 2.06/1.18 $\simeq 1.75$ larger than critical Bonnor-Ebert mass for the same gas temperature at the boundary $\left(M_{\mathrm{BE}} \simeq 2.6 M_{\odot}\right.$ for $T_{\mathrm{g}}=10 \mathrm{~K}$, see Eq. (1)).
Table 3. Critical mass and density contrast as function of polytropic index.

\begin{tabular}{lll}
\hline \hline$n$ & $\alpha_{n}$ & $\rho_{\mathrm{c}} / \rho_{\mathrm{b}}$ \\
\hline 3.5 & 3.37 & 88 \\
4 & 2.85 & 50 \\
5 & 2.33 & 32 \\
10 & 1.64 & 19 \\
20 & 1.39 & 15 \\
$\infty$ & 1.18 & 14 \\
\hline
\end{tabular}

\section{Discussion and conclusions}

We have examined the gas temperature distribution to be expected in interstellar pre-protostellar cores heated by the external ISRF. We find that when (as in observed cores), the central density exceeds $3 \times 10^{4} \mathrm{~cm}^{-3}$, there is coupling between the gas and dust temperatures and hence the gas temperature (like the dust) decreases with decreasing radius. At larger radii and smaller densities, the dust and gas decouple and the gas temperature may (for low external pressures as in the Taurus cloud) decrease towards the values expected for heating by galactic cosmic rays of around $10 \mathrm{~K}$. The region where gas and dust temperatures are coupled is somewhat interior to the region where $\mathrm{CO}$ is highly depleted. However, $\mathrm{CO}$ depletion does not seem greatly to affect the temperature distribution mainly because cooling by gas-grain collisions becomes dominant.

The observed values of the temperature of around $10 \mathrm{~K}$ in many pre-stellar cores allow limits to be placed on the cosmic ray ionization rate similar to the standard value of order $10^{-17} \mathrm{~s}^{-1}$ based on the measured cosmic ray flux. The fact that measured gas temperatures in cores show so little spatial variation (Benson \& Myers 1989; Tafalla et al. 2002) suggests to us that the observed thermometers mainly trace layers of moderate depletion where gas-grain coupling is not playing an important role.

We have also examined the consequences of such a temperature distribution for the density distribution in hydrostatic equilibrium cores. The changes caused by the "real temperature distribution" are minor and the characteristics of a "marginally stable Bonnor-Ebert sphere" are similar to those in the isothermal case.

An interesting point which emerges from these calculations is that the temperature in the core nucleus is sensitive to the external radiation field (because the core nucleus as a rule is heated by small particle MIR emission from the borders of the surrounding cloud). This is in particular the case for cores with high external pressure (and hence high density) such as those studied by Johnstone et al. $(2001,2000)$ in the Orion B and $\rho$ Oph clouds where $p_{\text {ext }}$ appears to be above $10^{6} \mathrm{~cm}^{-3} \mathrm{~K}$. In these cases, we expect the dust temperature to follow the gas temperature and thus the behavior should be roughly like a negative index polytrope. The external radiation fields are also higher in Orion and Ophiuchus than in Taurus and thus the core masses may also rise somewhat. For constant external pressure, the mass of the marginally stable Bonnor-Ebert sphere rises with about the 0.35 power of the external radiation 
field. It would be useful to have direct temperature estimates to confirm these expectations.

Another result of this study is that high mass thermally supported cores are incompatible with high column density. Thus it is difficult to imagine observed high column density (greater than $10^{22} \mathrm{~cm}^{-2}$ ) high mass (greater than $10 M_{\odot}$ ) cores going through a series of quasi-equilibrium states en route to collapse. Hence we suspect that higher mass cores are either not stable or have other (magnetic) means of support. These other means of support become apparent observationally both because observed cores show large departures from spherical symmetry and also because the observed line widths in most cores can only be explained in terms of turbulence and often of supersonic turbulence.

Thus thermally supported cores may be the exception rather than the rule. It is significant nonetheless that cores in regions such as Taurus where predominantly low mass star formation is taking place have in general line profiles showing a large component of thermal broadening. This suggests that where (as in Taurus), the pivotal state may be a core with predominantly thermal support, only low mass stars are likely to form. In clouds such as Ophiuchus and Orion with higher radiation fields and pressures and with cores having predominantly nonthermal support, higher mass stars may become possible and star formation may take another course.

It is also significant that occasionally, one finds cases like B68 where the data are consistent with hydrostatic equilibrium (marginally stable) and pure thermal support. In fact, we find that when one takes the gas temperature dependence into account, B68 is marginally stable. But irrespective of whether this is true or not, B68 gives every sign of being close to the pivotal state from which protostellar collapse will commence.

Acknowledgements. It is a pleasure to thank A. Natta and P. Caselli for constructive criticism and Jens Kauffman for useful comments on the manuscript. We acknowledge financial support from the EC Research Training Network "The Formation and Evolution of Young Stellar Clusters" and grant COFIN-2000. JG acknowledges support from scolarship SFRH/BD/6108/2001 awarded by the Portuguese Ministry of Education.

\section{References}

Alves, J. F., Lada, C. J., \& Lada, E. A. 2001, Nature, 409, 159 Bacmann, A., André, P., Puget, J. L., et al. 2000, A\&A, 361, 558

Bakes, E. L. O., \& Tielens, A. G. G. M. 1994, ApJ, 427, 822

Barranco, J. A., \& Goodman, A. A. 1998, ApJ, 504, 207

Benson, P. J., \& Myers, P. C. 1989, ApJS, 71, 89

Bourke, T. L., Hyland, A. R., Robinson, G., James, S. D., \& Wright, C. M. 1995, MNRAS, 276, 1067

Bergin, E. A., Alves, J., Huard, T., \& Lada, C. J. 2002, ApJ, 570, L101

Black, J. H. 1994, in The First Symposium on the Infrared Cyrrus and Diffuse Interstellar Clouds, ed. R. M. Cutri, \& W. B. Latter, ASP Conf. Ser., 58, 355

Boland, W., \& de Jong, T. 1984, A\&A, 134, 87
Bonnor, W. B. 1956, MNRAS, 116, 351

Boulanger, F., \& Perault, M. 1988, A\&A, 330, 964

Boulanger, F., Reach, W. T., et al. 1996, A\&A, 315, L325

Burke, J. R., \& Hollenbach, D. J. 1983, ApJ, 265, 223

Caselli, P., Walmsley, C. M., \& Zucconi, A., et al. 2002a, ApJ, 565, 331

Caselli, P., Walmsley, C. M., \& Zucconi, et al. 2002b, ApJ, 565, 344

Chièze, J.-P. \& Pineau des Fôrets, G. 1987, A\&A, 183, 98

Crutcher, R. M. 1999, ApJ, 520, 706

de Jong, T., Dalgarno, A., \& Boland, W. 1980, A\&A, 91, 68

Dickman, R. L., \& Clemens, D. P. 1983, ApJ, 271, 143

Ebert, R. 1955, Z. für Astr., 37, 217

Ebert, R. 1957, Z. für Astr., 42, 263

Evans, N. J., Rawlings, J. M. C., Shirley, Y., \& Mundy, L. G. 2001, ApJ, 557, 193

Falgarone, \& E., Puget, J. L. 1985, A\&A, 142, 157

Flannery, B. P., Roberge, W., \& Rybicki, G. B. 1980, ApJ, 236, 598

Goldsmith, P. F. 2001, ApJ, 557, 736

Goodman, A. A., Barranco, J. A., Wilner, D. J., \& Heyer, M. H. 1998, ApJ, 504, 223

Habing, H. J. 1968, Bull. Astron. Inst. Netherlands, 19, 421

Harvey, D. W. A., Wilner, D. J., Lada, C. J., \& Myers, P. C. 2001, ApJ, 2001, 563

Hasegawa, T. I. 1988, PASJ, 40, 219

Horedt, G. P. 2000, PASJ, 52, 217

Johnstone, D., Wilson, C. D., Moriarty-Schieven, G., et al. 2000 , ApJ, 545,327

Johnstone, D., Fich, M., Mitchell, G. F., \& Moriarty-Schieven, G. 2001, ApJ, 559, 307

Kramer, C., Alves, J., Lada, C. J., et al. 1999, A\&A, 342, 257

Krügel, E., \& Walmsley, C. M. 1984, A\&A, 130, 5

Lada, C. J., Lada, E. A., Bally, J., \& Clemens, D. 1994, ApJ, 429, 694

Lombardi, M., \& Bertin, G. 2001, A\&A, 375, 1091

McCrea, W. H. 1957, MNRAS, 117, 562

McKee, C. F., Zweibel, E. G., Goodman, A. A., \& Heiles, C. 1993, in Protostars \& Planets III, ed. E. H. Levy, \& J. I. Lunine (Tucson: The University of Arizona Press), 327

McKee, C. F. 1999, in The origin of stars and planetary systems, ed. C. J. Lada, \& N. D. Kylafis (Kluwer, NATO Sci. Ser. 540), 29

McLaughlin, D. E., \& Pudritz, R. E. 1996, ApJ, 469, 194

Maloney, P. 1988, ApJ, 334, 761

Mouschovias, T. Ch., \& Spitzer, L. 1976, ApJ, 210, 326

Mathis, J. S., Mezger, P., \& Panagia, N. 1983, A\&A, 128, 212

Myers, P. C., \& Goodman, A. A. 1988a, ApJ, 326, L27

Myers, P. C., \& Goodman, A. A. 1988b, ApJ, 329, 392

Myers, P. C., Ladd, E. F., \& Fuller, G. A. 1991, ApJ, 372, L95

Shu, F. H., Adams, F. C., \& Lizano, S. 1987, ARA\&A, 25, 31

Tafalla, M., Myers, P. C., Caselli, P., \& Walmsley, et al. 2002, ApJ, in press

Tassoul, J.-L. 1978, Theory of rotating Stars (Princeton: University Press)

Tomisaka, K., Ikeuchi, S., \& Nakamura, T. 1988, ApJ, 335, 239

Ward-Thompson, D., Motte, F., \& André, P. 1999, MNRAS, 305, 143

Ward-Thompson, D., André, P., \& Kirk, J. M. 2001, MNRAS, 329, 257

Zeldovich, Ya. B. 1963, Voprosi Kosmogonii (USSR), 9, 157

Zucconi, A., Walmsley, C. M., \& Galli, D. 2001, A\&A, 376, 650 